\title{
Utilização de gestão do conhecimento por meio de ferramentas de groupware e educação à distância
}

\author{
José Gilson de Almeida Teixeira Filho', Maria Augusta Ferreira Lopes², \\ Ivaldir Honório de Farias Júnior ${ }^{3}$ \\ ${ }^{1}$ Universidade de Pernambuco (UPE) \\ Recife - PE - Brasil \\ ${ }^{2}$ UniFAE Centro Universitário \\ Caixa Postal 80230080 - Curitiba - PR - Brasil \\ ${ }^{3}$ Universidade Federal de Pernambuco (UFPE) \\ Recife - PE - Brasil \\ jgatf@upe.poli.br, augustalopes@gmail.com, ihfj@cin.ufpe.br
}

\begin{abstract}
Ahead of all the challenges that the technology backwards currently, is each time more necessary to share and to take information to a bigger number of people. In this article it is presented one another vision based on techniques and methodologies of groupware and education in the distance as a facility for the application of the management of the knowledge, since these are tools sufficiently used currently for great companies, institutions of superior education and government to try to increase the communication and contribution enters the participant members of these organizations.
\end{abstract}

Resumo. Diante de todos os desafios que a tecnologia trás atualmente, fica cada vez mais necessário compartilhar e levar informações a um maior número de pessoas. Neste artigo é apresentada outra visão baseada em técnicas e metodologias de groupware e educação a distância como um facilitador para a aplicação da gestão do conhecimento, visto que essas são ferramentas bastante utilizadas atualmente por grandes empresas, instituições de ensino superior e governo para tentar aumentar a comunicação $e$ colaboração entre os membros participantes dessas organizações.

\section{Introdução}

A educação a distancia (EAD), ou e-learning, incentiva a troca de experiências, utilizando novos métodos e tecnologias como mecanismo de aprendizado contínuo e abertura de novos horizontes. Apesar do vasto crescimento de ferramentas para esta área, ainda hoje, existem grandes barreiras que precisam ser superadas como principalmente no tocante a visão e receio dos professores em utilizar tais tecnologias.

O groupware possui três aspectos que são fundamentais para iteração entre pessoas, e entre grupos de pessoas de diversas áreas de atuação, são eles: comunicação, coordenação e colaboração. Podemos observar também o CSCW (computer supported cooperative work), ou seja, trabalho cooperativo com auxílio de computador onde o interesse principal é em estudar como os grupos trabalham, e procurar descobrir novas tecnologias que auxiliem esses trabalhos em grupo. 
Para aplicar o groupware e a EAD nada melhor do que utilizar os conceitos de gestão do conhecimento que tem como objetivo principal favorecer o aprendizado coletivo e o desenvolvimento de organizações estruturadas a partir de requisitos que se tornam cada vez mais sólidos neste novo tempo denominado de era do conhecimento.

Este artigo tem como objetivo apresentar uma introdução aos conceitos, tecnologias e metodologias da área de educação à distância e gestão do conhecimento.

\section{Educação à Distância (EAD)}

Diversos autores definiram educação à distância, adotando diferentes pontos de vista. Estas definições evoluíram juntamente com os novos conhecimentos e transformações da sociedade. Abaixo serão apresentadas as definições dadas por alguns destes autores.

Educação a distância é o processo de ensino-aprendizagem, mediado por tecnologias, onde professores e alunos estão separados espacial e/ou temporalmente (MORAN, 2000).

O termo educação a distância cobre as distintas formas de estudo em todos os níveis, que não se encontram sob a contínua, imediata supervisão de professores presentes com os estudantes em aula, mas que são beneficiados por um planejamento, guia e acompanhamento de uma organização educativa, incluindo todos os métodos de ensino, tendo as fases interativa e proativa do ensino realizando-se através de meios distintos. (HOLMBERG, 1985 apud EMERENCIANO, WICKERT 1998).

O ensino a distância é um sistema tecnológico de comunicação bidirecional, que pode ser massivo e que substitui a interação pessoal, na sala de aula, de professor e aluno, como meio preferencial de ensino, pela ação sistemática e conjunta de diversos recursos didáticos e pelo apoio de uma organização e tutoria que propiciam a aprendizagem independente e flexível dos alunos (ARETIO, 1992 apud REZENDE, 2000).

Segundo Muirhead (1999) alguns aspectos são importantes de serem observados na definição de Educação a Distância:

- Professor e o aluno devem estar separados durante a maior parte do curso;

- A influência de uma organização, incluindo um sistema de avaliação do aluno;

- Uso de meios educacionais para unir o professor e o aluno para que este tenha sucesso;

- Oferecimento de uma comunicação bidirecional entre professor, aluno, ou agente educacional e aluno.

De acordo com Resende (2000), a expressão ensino está ligada às atividades de treinamento, instrução e socialização da informação, enquanto Educação relaciona-se ao processo de formação humana que implica aprender, criar, construir conhecimento, inovar, participar ativamente do seu próprio crescimento.

Fainhato (2000) considera que EAD deve possuir as seguintes características mais relevantes para obter um alto grau de qualidade:

- Ser considerada como um sistema educacional dinâmico composto por subsistemas que interagem e que representam uma estrutura gerencial e pedagógica a ser realizada. Como tal deve adotar critérios de eficiência (desempenho interno, 
técnico), eficácia (desempenho externo), efetividade (eqüidade e desenvolvimento econômico e social) e relevância/significado social;

- Caracterizar-se pela distância física e/ou temporal entre professor e aluno, onde aquele se faz presente através do planejamento e do diálogo contínuo com o aprendiz durante o curso;

- Adotar princípios e valores éticos, políticos e pedagógicos visando à formação do homem como ser integral inserido em um contexto social;

- Ser rigorosa em sua concepção e planejamento, pois é influenciada por fatores contingenciais como a falta de controle imediato do processo e do aprendiz;

- Utilizar-se da tecnologia (termo definido a seguir) para mediar o processo ensinoaprendizagem e a interatividade entre professor e aprendiz;

- Valorizar a interação e a comunicação entre todos os participantes do processo;

- Enfatizar o papel do professor como orientador (tutor) do aprendiz e em constante interatividade com ele;

- O aluno como centro do processo, autodidata, aprendendo a aprender, a pensar e a criar, respeitando-se seu tempo, ritmo e método de aprendizagem;

- Trazer consigo o conceito de educação continuada, num processo de formação permanente.

A educação à distância, antes vista com preocupação, hoje assume um papel importante na tentativa de viabilizar a democratização e a universalização do ensino. Não que esta vá substituir o ensino presencial, mas, com certeza, é um caminho a ser considerado.

\subsection{Os desafios da expansão acelerada da EAD no Brasil}

Para melhor analisar uma questão, é necessário observar a dimensão de forma maior. No caso da Educação à Distância, o primeiro desafio a ser enfrentado é que esta modalidade de ensino a tempos vem sendo marginalizada até bem pouco tempo pelos órgãos oficiais e pelas próprias IES (Instituições de Ensino Superior). Ainda é superficial a quantidade e qualidade dos dados sobre a presença da EAD no sistema de educação superior. Hoje, já se têm algumas fontes de observação da situação da EAD no Brasil como as citadas a seguir:

O Anuário Brasileiro Estatístico de Educação Aberta e à Distância - ABRAEAD (SANCHEZ, 2005) contendo documentos disponibilizados pelo portal do MEC. Esse anuário parece ser inicialmente, uma fonte de dados bastante completa na medida em que seu objetivo é, justamente, dar uma visão dos "números da educação à distância no Brasil". Em outros casos, a descrição ainda é pobre e restrita ao ano de 2004 e à amostra das 62 instituições participantes da investigação, quando seria importante ter disponível um conjunto de dados das IES e séries estatísticas.

A segunda fonte de informações consiste nos dados oriundos do MEC. Pode-se encontrar documentos contendo a relação de IES credenciadas para ministrar cursos de graduação e de especialização à distância (IPAE, 2007). O MEC regulamentou recentemente o uso de $20 \%$ da carga-horária para atividades à distância (MORAN; ARAUJO FILHO; SIDERICOUDES, 2007).

Uma terceira alternativa é consultar diretamente dos sites de algumas das IES relacionadas pelo MEC, pois nem tudo está contemplado nas duas primeiras fontes 
citadas. Algumas IES ainda possuem seu processo na modalidade semipresencial em disciplinas das grades curriculares dos cursos presenciais.

\section{Tecnologia e ferramentas de groupware}

O advento do desenvolvimento tecnológico e a necessidade de lidar com a complexidade e o tamanho das tarefas nas organizações, fez exigir maior interação entre as pessoas, e entre grupos de pessoas de diversas áreas de atuação. Proporcionar o suporte computacional para que pessoas possam interagir cooperativamente é o objetivo da disciplina que se convencionou chamar CSCW (Computer Supported Cooperative Work), e groupware tem sido usado para designar os softwares e hardwares que implementam essa tecnologia (BORGES, 1995).

CSCW é o acrônimo para computer supported cooperative work (trabalho cooperativo com auxílio de computador), e foi cunhado por Irene Greif e Paul Cashmann em 1984, ao organizarem um workshop com pessoas de várias disciplinas que compartilhavam o interesse em estudar como grupos trabalham, e procurar descobrir meios como a tecnologia (especialmente computadores) poderia auxiliá-los (BANNON e SCHMIDT, 1993).

Ellis (1993) complementa que o objetivo de groupware é dar assistência aos grupos na comunicação, colaboração e coordenação de suas atividades. Alguns autores definem groupware especificamente como "sistemas de computadores que assistem grupos de pessoas engajadas em uma tarefa (ou objetivo) comum e que provêem uma interface com um ambiente compartilhado".

Numa visão ainda mais prática, Hills (1997) considera que groupware é qualquer ferramenta que ajuda as pessoas a trabalharem juntas mais fácil e eficazmente, citando o telefone como a mais simples e comumente utilizada dessas ferramentas, que permitem realizar três funções básicas:

- Comunicação - Ajudar pessoas a compartilhar informações;

- Coordenação - Ajudar pessoas a coordenar seus papéis pessoais com os outros;

- Colaboração - Ajudar pessoas a trabalharem juntas.

Para realizar tais propósitos, o sistema de groupware deve permitir que duas ou mais pessoas trabalhem juntas ajudando a compartilhar seus conhecimentos e especialidades, automatizar suas atividades, criar uma memória organizacional, e a conectá-las, mesmo através de pontos geográficos e tempos diferentes, podendo ser visualizada em uma matriz espaço-temporal como a seguir na tabela 1.

Tabela 1 - Taxonomia espaço-temporal do groupware (JOHANSEN, 1991)

\begin{tabular}{c|c|c}
\hline Taxonomia espaço-temporal & Mesmo tempo & Tempo diferente \\
\hline Mesmo local & $\begin{array}{c}\text { Interação face a } \\
\text { face }\end{array}$ & $\begin{array}{c}\text { Interação } \\
\text { assíncrona }\end{array}$ \\
\hline Local diferente & $\begin{array}{c}\text { Interação } \\
\text { distribuída } \\
\text { síncrona }\end{array}$ & $\begin{array}{c}\text { Interação } \\
\text { distribuída } \\
\text { assíncrona }\end{array}$ \\
\hline
\end{tabular}


Os sistemas de CSCW normalmente são classificados de acordo com essa matriz de localização/tempo, utilizando a distinção entre trabalho realizado ao mesmo tempo (síncrono), ou em tempos diferentes (assíncrono), e no mesmo local (face a face), ou em locais diferentes (distribuído), como informa Johansen (1991).

\section{Gestão do Conhecimento}

Apesar do termo gestão e conhecimento serem usados largamente na literatura acadêmica de forma isolada, só nos últimos anos a expressão gestão do conhecimento veio a ser utilizada de forma mais acentuada. Tal expressão abarca um largo espectro de significados, não tendo um consenso conceitual, chegando mesmo a significações contraditórias. A gestão do conhecimento fomentou a ênfase no capital intelectual e reacendeu a importância do indivíduo na competitividade organizacional, vindo ancorar a reformulação do antigo modelo organizacional para o modelo contemporâneo, cuja fundamentação é construída no conceito de organização baseada em conhecimento. A gestão do conhecimento possibilita um incrementalismo lógico segundo Mintzberg (2000) facilitando o surgimento de estratégias emergentes, diminuindo a incerteza, ou seja, é a partir da gestão do conhecimento que a empresa possibilita fluxos de informações mais eficientes e respostas mais rápidas.

Nonaka e Takeuchi (1997) definem a gestão do conhecimento como o processo sistemático de identificação, criação, renovação e aplicação dos conhecimentos que são estratégicos na vida de uma organização.

Entende-se por conhecimento a informação interpretada, ou seja, o que cada informação significa e que impactos no meio cada informação pode causar, de modo que a informação possa ser utilizada para importantes ações e tomadas de decisões. Sabendo como o meio reage às informações, pode-se antever as mudanças e se posicionar de forma a obter vantagens e ser bem sucedido nos objetivos a que se propõe (WIKIPÉDIA, 2007).

Gestão do conhecimento significa organizar as principais políticas, processos e ferramentas gerenciais e tecnológicas à luz de uma melhor compreensão dos processos de geração, identificação, validação, disseminação, compartilhamento e uso dos conhecimentos estratégicos para gerar resultados (econômicos) para a empresa e benefícios para os colaboradores (TERRA, 2001).

Segundo Stewart (2002), gestão do conhecimento corresponde a identificar o que se sabe, captar e organizar esse conhecimento e utilizá-lo de modo a gerar retornos. O autor explica que o termo gestão do conhecimento é recente no meio organizacional, tanto que pesquisa na internet pelo termo gestão do conhecimento em 1996 traria como resposta apenas umas dezenas de resultados. Em 2005, tal pesquisa apresentava 842.000 (oitocentos e quarenta e dois mil) termos como resposta e atualmente apresenta aproximadamente 4.510 .000 (quatro milhões e quinhentos e dez mil) respostas no Google, um dos mecanismos de busca mais usados do mundo (GOOGLE, 2007).

Para implementação de um processo de gestão do conhecimento em uma organização, deve-se atentar para três dimensões condicionantes de uma organização do conhecimento: infra-estrutura organizacional, pessoas e tecnologia - visando ao alcance dos objetivos individuais e organizacionais. Tais dimensões podem ser apresentadas em um modelo proposto por Angeloni (2002), mostrado na figura 1. O modelo foi 
construído sob a forma de átomo para que se possa compreender o dinamismo das interações das variáveis organizacionais, onde nos orbitais encontram-se as dimensões e, no núcleo, a empresa da era do conhecimento.

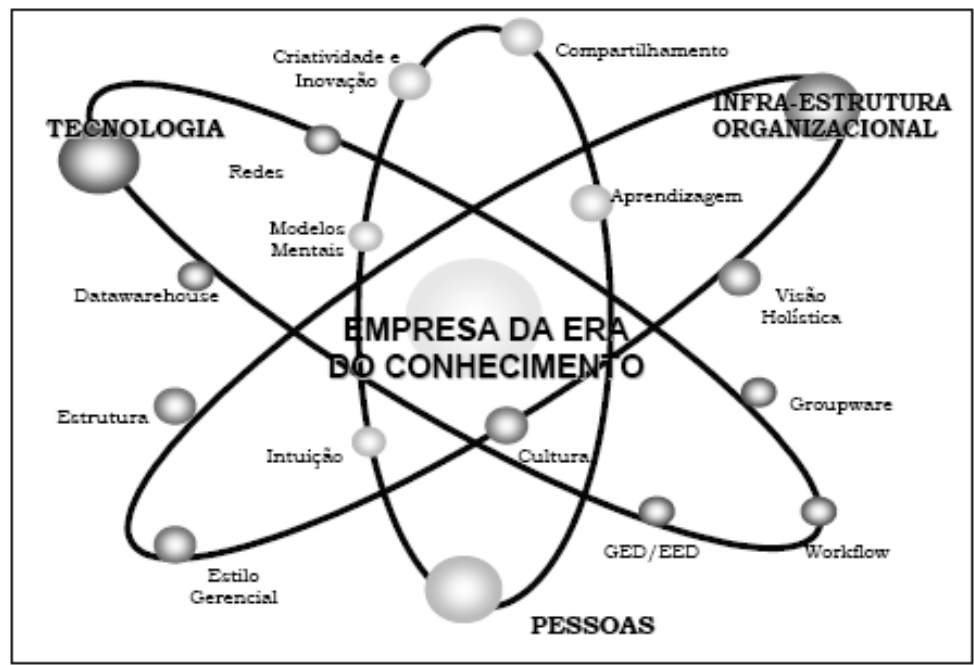

Figura 1 - Modelo de Organização de Conhecimento (ANGELONI, 2002)

O modelo a seguir, baseado em Terra (2001), explicita as dimensões da prática gerencial, relacionadas à gestão do conhecimento, baseadas em 7 dimensões conforme a figura 2 a seguir:

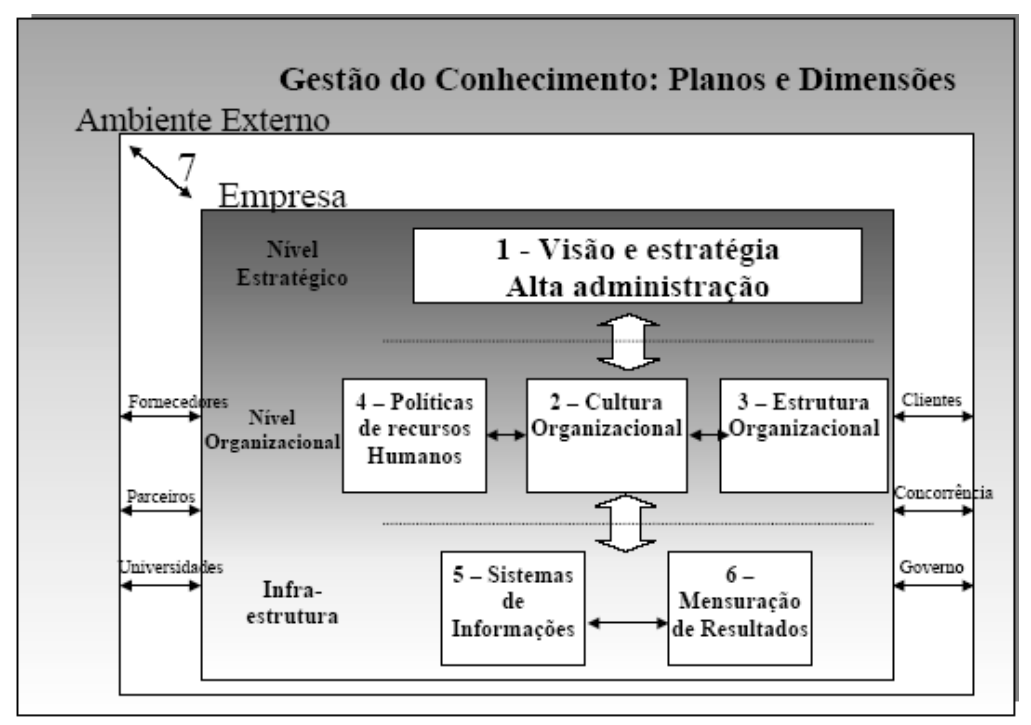

Figura 2 - Modelo conceitual sobre gestão de conhecimento na empresa (TERRA, 2001)

O modelo almeja mostrar que a gestão do conhecimento não está atrelada a apenas um dos aspectos que envolvem a organização, mas, pelo contrário, está vinculada a diversos fatores e atores organizacionais, revelando, assim, sua complexidade. 


\section{Aplicação da gestão do conhecimento utilizando ferramentas para groupware e EAD}

A gestão do conhecimento surge, de certa maneira, como criatura da tecnologia, apesar de na definição de gestão do conhecimento não dizer explicitamente a necessidade de um sistema computacional, defende Stewart (2002). A tecnologia da informação (TI) é uma das ferramentas que possibilitam a implantação da gestão do conhecimento utilizando ferramentas como groupware e EAD, considerado um importante instrumento para armazenar e recuperar informações; a TI, entretanto, não deve ser confundida com a gestão do conhecimento propriamente dita, mas sim como uma ferramenta de apoio que permitirá a geração, o armazenamento, o controle e a difusão do conhecimento.

Na prática, o que ocorre nas empresas que implementam uma gestão do conhecimento é a construção de um conjunto de aplicativos capacitadores para o compartilhamento de conhecimento que abrangem o seguinte: intranet, tecnologia groupware, armazenamento e busca de dados, sistemas e aplicativos de apoio à decisão, fontes de informações online, entre outros, sendo enfatizadas tais tecnologias, em detrimento das pessoas (RODRIGUES FILHO, 2002).

Segundo Stewart (2002) o fato de tais tecnologias serem utilizadas como ferramentas de apoio e facilitadoras à gestão do conhecimento têm levado muitas organizações a colocarem nas mãos do departamento de TI a implantação de programas de gestão do conhecimento, em detrimento do departamento de Recursos Humanos.

Bhatt (2004) e Swan (2000) comentam que a parcela habilitadora do ferramental tecnológico para a gestão do conhecimento é bem menor do que aspectos relativos à cultura, a pessoas e a processos organizacionais, observando o limite da contribuição da TI como direcionador de um programa de gestão do conhecimento mostrado na figura 3 a seguir.

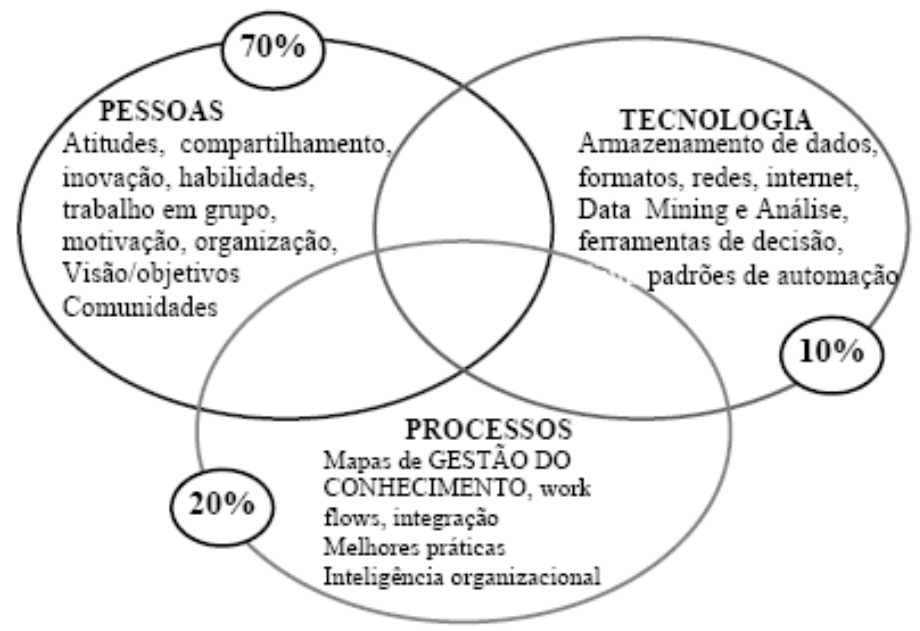

Figura 3 - Gestão do conhecimento: componentes e sub-elementos (BHATT, 2004)

Conforme figura 3, a implementação de um programa de gestão do conhecimento na empresa deve focar, essencialmente, e estar muito mais voltada para as pessoas, com $70 \%$ de participação, depois com processos administrativos, com 
$20 \% \%$, e, finalmente com o suporte a TI, que participa apenas com $10 \%$ para o suporte e eficácia do programa.

\subsection{Aplicação prática dos conceitos em uma ferramenta gratuita}

Surge em 2001 e o Moodle (acrônimo de Modular Object-Oriented Dynamic Learning) é um software livre (open source), de apoio à aprendizagem, executado num ambiente virtual e também conhecido como um Learning Management System, ou seja, um Sistema de Gestão de Aprendizagem em trabalho colaborativo. É um dos softwares mais usados atualmente nesse contexto, é gratuito e pode ser instalado em diversos ambientes (Unix, Linux, Windows, etc.). Criado na linguagem de programação PHP, usa vários tipos de bancos de dados desde o MySQL até o Oracle. Já existe também uma versão traduzia para o português e outros 60 idiomas.

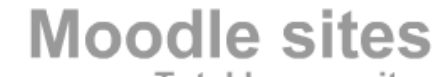

Total known sites

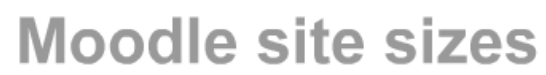

Number of users per site
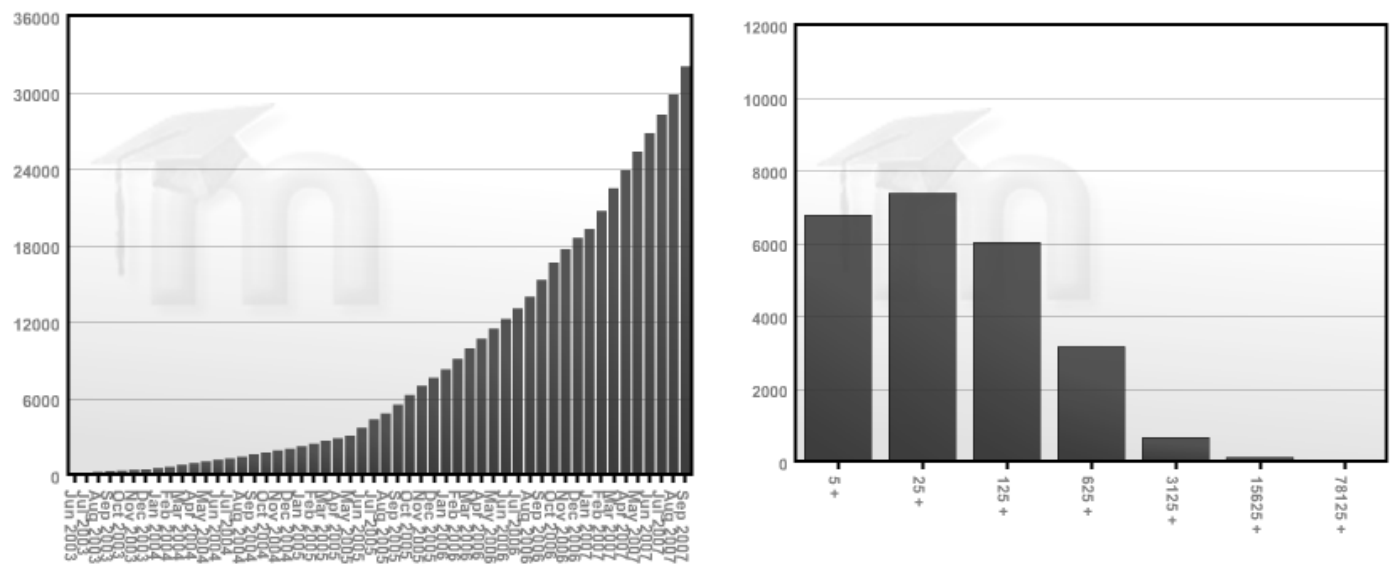

Figura 4 - Estatísticas do Moodle: Sites Registrados: 34.741; Cursos:

1.447.801; Usuários: 14.632.299; Professores: 1.744.245. Existem 75 sites com mais de 20 mil usuários. 0 site com a maioria dos usuários é o próprio Moodle com 43 cursos e 326.349 usuários inscritos.

Muitas escolas, centros de formação em níveis técnicos e superiores estão adotando essa ferramenta com sucesso, principalmente porque o conteúdo é desenvolvido colaborativamente por uma comunidade virtual que reúne desde programadores de softwares livres, passando por administradores de sistema, professores, alunos, funcionários e outros tipos de usuários de todo o mundo.

Principais características encontradas nessa ferramenta: Fóruns, Gestão de conteúdos e conhecimento, aplicação de questionários e pesquisas com diversos formatos, blogs, wikis para colaboração de conhecimento, chat, glossários, suporte multi-idioma (MOODLE, 2007).

\section{Conclusão}

A abordagem da gestão do conhecimento está preocupada com a representação, organização, aquisição, criação, uso e evolução do conhecimento em várias formas. Verificou-se que ela é uma área multidisciplinar que encara o capital intelectual como um ativo gerenciável, envolvendo os recursos humanos, a organização e a cultura da organização, a tecnologia de informação, e os métodos e ferramentas que a suportam e a 
viabilizam. Também fica evidente que o processo de conversão do conhecimento é algo dinâmico que exige envolvimento de todos, numa visão compartilhada, em que todos precisam aprender e compartilhar os conhecimentos para promover o desenvolvimento da organização.

Dessa maneira percebemos a grande utilidade de ferramentas como groupware e educação à distância (EAD) para tornar essa evolução do conhecimento cada vez mais sólida principalmente num mundo globalizado onde temos a informação como chave para o sucesso tanto das pessoas como das organizações. Diante desse cenário muitas empresas podem iniciar uma transição da era da informação para era do conhecimento de forma mais rápida e acessível, principalmente através do uso de novas tecnologias e ferramentas que estão sendo disponibilizadas dia a dia por comunidades de voluntários espalhadas ao redor do mundo.

\section{Referências}

ANGELONI, Maria T. (2002) - Organizações do conhecimento: infra-estrutura, pessoas e tecnologia. São Paulo: Saraiva.

BANNON, L.J., SCHMIDT, K. (1993) - CSCW: Four Characters in Search of a Context. In: Readings in Groupware and Computer-Supported Cooperative Work, p. 50-56. San Mateo: Morgan Kaufmann.

BHATT, D. Excellence Model and Knowledge Management Implications. [online]. Disponível na Internet via www. URL: http://www.eknowledgecenter.com/articles/1010/1010.htm. Acesso em: 14/07/2004.

BORGES, M.R.S., CAVALCANTI M.C.R., MACHADO, L. (1995) - Suporte por Computador ao Trabalho Cooperativo. XV Congresso da Sociedade Brasileira de Computação, - XIV Jornada de Atualização em Informática, Canela.

ELLIS, C. A., GIBBS S., REIN, G.L. (1993) - Groupware: Some Issues and Experiences. Communications of the ACM/January 1991/Vol.34.n.1. In: Readings in groupware and computer-supported cooperative work. San Mateo: Morgan Kaufmann, p. 9-28.

EMERENCIANO, Maria do Socorro Jordão e WICKERT Maria. L. Scarpini. Conceituação de educação a distância. Curso de pós-graduação em Educação a Distância da Universidade Católica de Brasília (UCB). Brasília, DF: Universa, 1998.

FAINHATO, Beatriz. (2000) - A qualidade da Educação a distância. Tradução de Maria Lucia Scarpini Wickert, para o curso de pós-graduação em educação a distância da Universidade Católica de Brasília (UCB).

GOOGLE. Site de busca de informações. Disponível em: http://www.google.com. Acesso em: 26 Out. 2007.

HILLS, Melanie. (1997) - Intranet as groupware.. Wiley Computer Publishing. USA.

IPAE. Instituto de Pesquisas Avançadas em Educação. Cadastro dos cursos de especialização. Jornal da Educação (online) Rio de Janeiro: Instituto de Pesquisas Avançadas em Educação. Ano 11 n. 2554, 20 jun. 2005. Disponível em: http://www.ipae.com.br. Acesso em: 25 Out. 2007. 
JOHANSEN, R.; SIBBET D.; BENSON S.; MARTIN A.; MITTMAN R e SAFFO P. (1991) - Leading business teams. Addison Wesley.

MINTZBERG, Henry. (2000) - Safári de estratégia. Rio de Janeiro: Campus.

MOODLE. Software livre de apoio à aprendizagem. Disponível em: http://www.moodle.org. Acesso em: 25 Out. 2007.

MORAN, J.M.; ARAUJO FILHO, M.; SIDERICOUDES, O. A ampliação dos vinte por cento a distância: estudo de caso da Faculdade Sumaré-SP. Trabalho apresentado no XII Congresso Internacional da Associação Brasileira de Educação à distância, Florianópolis, $20 \quad$ set. $2005 . \quad$ Disponível em: http://www.abed.org.br/congresso2005/por/pdf/172tcc3.pdf . Acesso em: 25 Out. 2007.

MORAN, José Manuel. (2000) - Mudanças na comunicação pessoal: Gerenciamento integrado da comunicação pessoal, social e tecnológica. São Paulo: Paulinas.

MUIRHEAD, Brent. (1999) - Attitudes Toward Interactivity in a Graduate Distance Education Program: A Qualitative Analysis. Dissertação (Phd.D.). Academic dissertation Publisher. Disponível em: $<$ http://ifets.massey.ac.nz/periodical/vol_1_2000/muirhead.html>. Acesso em: 25/10/2007.

NONAKA, I.; TAKEUCHI, H. (1997) - Criação de conhecimento na empresa: como as empresas japonesas geram a dinâmica da inovação. Rio de Janeiro: Campus.

REZENDE, Flavia. (2000) - Tecnologia e Educação. Curso de Pós-graduação em Docência do Ensino Superior. Rio de Janeiro: CEP/UFRJ.

RODRIGUES FILHO, J; OLIVEIRA, R.R.; TORRES, M.F. (2002) - Gestão do Conhecimento e as Transformações no Setor Elétrico: Um esboço para o estudo da CHESF. Anais do Congresso Anual da Sociedade Brasileira de Gestão do Conhecimento, São Paulo.

SANCHEZ, F. (Coord.). Anuário brasileiro estatístico de educação aberta e à distância. ABRAEAD-2005. São Paulo: Instituto Monitor, 2005

SCARBROUGH, H., SWAN, J., HISLOP, D. (2000) - Intranets and Knowledge Management: De-centred Technologies and the Limits of Technological Discourse. In: PRICHARD, C. HULL, R. CHUMER, M., WILLMOTT, H. (Editors), Managing Knowledge: Critical Investigations of Work and Learning. London: MacMillan.

STEWART, Thomas A. (2002) - A riqueza do conhecimento: o capital intelectual e a organização do século XXI. Rio de Janeiro. Campus.

TERRA, J. C. C.. (2001) - Gestão do conhecimento: aspectos conceituais e estudo exploratório sobre as práticas de empresas brasileiras. In: FLEURY, Maria T. L. e OLIVEIRA JR, Moacir de Miranda. Gestão estratégica do conhecimento: integrando aprendizagem, conhecimento e competências. São Paulo: Atlas.

WIKIPÉDIA. Enciclopédia livre e fonte de informação. Disponível em: http://pt.wikipedia.org/wiki/Gest\%C3\%A3o_do_conhecimento. Acesso em: 03 Out. 2007. 